\title{
The Natural Operators Similar to the Twisted Courant Bracket One
}

\author{
Włodzimierz M. Mikulskiø
}

\begin{abstract}
Given natural numbers $m \geq 3$ and $p \geq 3$, all $\mathcal{M} f_{m}$-natural operators $A_{H}$ sending $p$-forms $H \in \Omega^{p}(M)$ on $m$-manifolds $M$ into bilinear operators $A_{H}:\left(\mathcal{X}(M) \oplus \Omega^{1}(M)\right) \times\left(\mathcal{X}(M) \oplus \Omega^{1}(M)\right) \rightarrow \mathcal{X}(M) \oplus \Omega^{1}(M)$ transforming pairs of couples of vector fields and 1-forms on $M$ into couples of vector fields and 1-forms on $M$ are founded. If $m \geq 3$ and $p \geq 3$, then that any (similar as above) $\mathcal{M} f_{m}$-natural operator $A$ which is defined only for closed $p$-forms $H$ can be extended uniquely to the one $A$ which is defined for all $p$-forms $H$ is observed. If $p=3$ and $m \geq 3$, all $\mathcal{M} f_{m}$-natural operators $A$ (as above) such that $A_{H}$ satisfies the Leibniz rule for all closed 3 -forms $H$ on $m$-manifolds $M$ are extracted. The twisted Courant bracket $[-,-]_{H}$ for all closed 3 -forms $H$ on $m$-manifolds $M$ gives the most important example of such $\mathcal{M} f_{m}$-natural operator $A$.
\end{abstract}

Mathematics Subject Classification. 58 A 99, 58 A 32.

Keywords. Natural operator, Twisted Courant bracket, Leibniz rule.

\section{Introduction}

The "doubled" tangent bundle $T \oplus T^{*}$ over $m$-dimensional manifolds $(m$ manifolds) is full of interest because it has the natural inner product, and the Courant bracket, see [1]. Besides, generalized complex structures are defined on $T \oplus T^{*}$, generalizing both (usual) complex and symplectic structures, see e.g. $[3,4]$.

In Sect. 2, the description from [2] of all $\mathcal{M} f_{m}$-natural bilinear operators

$$
A:\left(\mathcal{X}(M) \oplus \Omega^{1}(M)\right) \times\left(\mathcal{X}(M) \oplus \Omega^{1}(M)\right) \rightarrow \mathcal{X}(M) \oplus \Omega^{1}(M),
$$

transforming pairs of couples of vector fields and 1-forms on $m$-manifolds $M$ into couples of vector fields and 1-forms on $M$ will be shortly cited. The most important example of such $\mathcal{M} f_{m}$-natural bilinear operator $A$ is given by the Courant bracket $[-,-]^{C}$, see Example 2.2. This Courant bracket was used in [1] to define the concept of Dirac structures being hybrid of both symplectic and Poisson structures. 
In Sect. 2 we also deduce that the "trivial" Lie algebroid ( $T M \oplus$ $\left.T^{*} M, 0,0\right)$ is the only $\mathcal{M} f_{m}$-natural Lie algebroid $(E M,[[-,-]], a)$ with $E M$ $:=T M \oplus T^{*} M$.

In Sect. 3, using essentially the results from [2], if $m \geq 3$ and $p \geq 3$, we find all $\mathcal{M} f_{m}$-natural operators $A$ sending $p$-forms $H \in \Omega^{p}(M)$ on $m$ manifolds $M$ into bilinear maps

$$
A_{H}:\left(\mathcal{X}(M) \oplus \Omega^{1}(M)\right) \times\left(\mathcal{X}(M) \oplus \Omega^{1}(M)\right) \rightarrow \mathcal{X}(M) \oplus \Omega^{1}(M) .
$$

The most important example of such $A$ is given by the $H$-twisted Courant bracket $[-,-]_{H}$ for all 3 -forms $H$ on $m$-manifolds $M$, see Example 3.2. Properties of $[-,-]_{H}$ (as the Leibniz rule for closed 3 -forms $H$ ) were used in $[7,8]$ to define the concept of exact Courant algebroid.

In Sect. 4, we observe that if $m \geq 3$ and $p \geq 3$, then any (similar as above) $\mathcal{M} f_{m}$-natural operator $A$ which is defined only for closed $p$-forms $H$ can be extended uniquely to the one $A$ which is defined for all $p$-forms $H$.

In Sect. 5 , if $p=3$ we extract all $\mathcal{M} f_{m}$-natural operators $A$ as above satisfying the Leibniz rule

$$
A_{H}\left(\rho_{1}, A_{H}\left(\rho_{2}, \rho_{3}\right)\right)=A_{H}\left(A_{H}\left(\rho_{1}, \rho_{2}\right), \rho_{3}\right)+A_{H}\left(\rho_{2}, A_{H}\left(\rho_{1}, \rho_{3}\right)\right),
$$

for any closed $H \in \Omega^{3}(M), \rho_{1}, \rho_{2}, \rho_{3} \in \mathcal{X}(M) \oplus \Omega^{1}(M)$ and $M \in \operatorname{obj}\left(\mathcal{M} f_{m}\right)$.

From now on, $\left(x^{i}\right)(i=1, \ldots, m)$ denote the usual coordinates on $\mathbf{R}^{m}$ and $\partial_{i}=\frac{\partial}{\partial x^{i}}$ are the canonical vector fields on $\mathbf{R}^{m}$.

All manifolds considered in this paper are assumed to be finite dimensional second countable Hausdorff without boundary and smooth (of class $\left.\mathcal{C}^{\infty}\right)$. Maps between manifolds are assumed to be smooth (of class $\mathcal{C}^{\infty}$ )

\section{The Natural Bilinear Operators Similar to the Courant Bracket}

The general concept of natural operators can be found in the fundamental monograph [5]. In the paper, we need two particular cases of natural operators presented in Definitions 2.1 (below) and 3.1 (in the next section).

Let $\mathcal{M} f_{m}$ be the category of $m$-dimensional $\mathcal{C}^{\infty}$ manifolds as objects and their immersions of class $\mathcal{C}^{\infty}$ as morphisms ( $\mathcal{M} f_{m}$-maps).

Definition 2.1. A natural (called also $\mathcal{M} f_{m}$-natural) operator $A$ sending pairs of couples of vector fields and 1-forms on $m$-manifolds $M$ into couples of vector fields and 1-forms on $M$ is a $\mathcal{M} f_{m}$-invariant family of operators (functions)

$$
A:\left(\mathcal{X}(M) \oplus \Omega^{1}(M)\right) \times\left(\mathcal{X}(M) \oplus \Omega^{1}(M)\right) \rightarrow \mathcal{X}(M) \oplus \Omega^{1}(M),
$$

for all $m$-manifolds $M$, where $\mathcal{X}(M) \oplus \Omega^{1}(M)$ is the vector space of couples $(X, \omega)$ of vector fields $X$ on $M$ and 1-forms $\omega$ on $M$. Such $\mathcal{M} f_{m}$-natural operator $A$ is called bilinear if $A$ is bilinear (i.e., $A\left(\rho^{1},-\right)$ and $A\left(-, \rho^{2}\right)$ are linear (over the field $\mathbf{R}$ of real numbers) functions $\mathcal{X}(M) \oplus \Omega^{1}(M) \rightarrow$ $\mathcal{X}(M) \oplus \Omega^{1}(M)$ for any fixed $\left.\rho^{1}, \rho^{2} \in \mathcal{X}(M) \oplus \Omega^{1}(M)\right)$ for any $m$-manifold $M$. Such $\mathcal{M} f_{m}$-natural operator $A$ is called skew-symmetric if $A$ is skewsymmetric for any $m$-manifold $M$. 
The $\mathcal{M} f_{m}$-invariance of $A$ means that if $\left(X^{1} \oplus \omega^{1}, X^{2} \oplus \omega^{2}\right)$ and $\left(\bar{X}^{1} \oplus\right.$ $\bar{\omega}^{1}, \bar{X}^{2} \oplus \bar{\omega}^{2}$ ) are $\varphi$-related by an $\mathcal{M} f_{m}$ map $\varphi: M \rightarrow \bar{M}$ (i.e., $\bar{X}^{i} \circ \varphi=$ $T \varphi \circ X^{i}$ and $\bar{\omega}^{i} \circ \varphi=T^{*} \varphi \circ \omega^{i}$ for $\left.i=1,2\right)$, then so are $A\left(X^{1} \oplus \omega^{1}, X^{2} \oplus \omega^{2}\right)$ and $A\left(\bar{X}^{1} \oplus \bar{\omega}^{1}, \bar{X}^{2} \oplus \bar{\omega}^{2}\right)$.

The most important example of such $\mathcal{M} f_{m}$-natural bilinear operator $A$ is given by the (skew-symmetric) Courant bracket $[-,-]^{C}$ for any $m$-manifold $M$.

Example 2.2. On the vector bundle $T M \oplus T^{*} M$ there exist canonical symmetric and skew-symmetric pairings

$$
\left\langle X^{1} \oplus \omega^{1}, X^{2} \oplus \omega^{2}\right\rangle_{ \pm}=\frac{1}{2}\left(i_{X^{2}} \omega^{1} \pm i_{X^{1}} \omega^{2}\right)
$$

for any $X^{1} \oplus \omega^{1}, X^{2} \oplus \omega^{2} \in \mathcal{X}(M) \oplus \Omega^{1}(M)$, where $i$ is the interior derivative. Further, the (skew-symmetric) Courant bracket is given by

$$
\begin{aligned}
& {\left[X^{1} \oplus \omega^{1}, X^{2} \oplus \omega^{2}\right]^{C}} \\
& \quad=\left[X^{1}, X^{2}\right] \oplus\left(\mathcal{L}_{X^{1}} \omega^{2}-\mathcal{L}_{X^{2}} \omega^{1}+\mathrm{d}\left\langle X^{1} \oplus \omega^{1}, X^{2} \oplus \omega^{2}\right\rangle_{-}\right)
\end{aligned}
$$

for any $X^{1} \oplus \omega^{1}, X^{2} \oplus \omega^{2} \in \mathcal{X}(M) \oplus \Omega^{1}(M)$, where $[-,-]$ is the usual bracket on vector fields, $\mathcal{L}$ is the Lie derivative and $\mathrm{d}$ is the exterior derivative.

Theorem 2.3 [2]. If $m \geq 2$, any $\mathcal{M} f_{m}$-natural bilinear operator $A$ in the sense of Definition 2.1 is of the form

$$
\begin{gathered}
A\left(\rho^{1}, \rho^{2}\right) \\
=a\left[X^{1}, X^{2}\right] \oplus\left(b_{1} \mathcal{L}_{X^{2}} \omega^{1}+b_{2} \mathcal{L}_{X^{1}} \omega^{2}+b_{3} \mathrm{~d}\left\langle\rho^{1}, \rho^{2}\right\rangle_{+}+b_{4} \mathrm{~d}\left\langle\rho^{1}, \rho^{2}\right\rangle_{-}\right)
\end{gathered}
$$

for (uniquely determined by $A$ ) real numbers $a, b_{1}, b_{2}, b_{3}, b_{4}$, where $\rho^{i}=X^{i} \oplus$ $\omega^{i} \in \mathcal{X}(M) \oplus \Omega^{1}(M)$ for $i=1,2$ are arbitrary, and where $\langle-,-\rangle_{+}$and $\langle-,-\rangle_{-}$are as in Example 2.2.

Corollary 2.4 [2]. If $m \geq 2$, any $\mathcal{M} f_{m}$-natural skew-symmetric bilinear operator $A$ in the sense of Definition 2.1 is of the form

$$
\begin{gathered}
A\left(X^{1} \oplus \omega^{1}, X^{2} \oplus \omega^{2}\right) \\
=a\left[X^{1}, X^{2}\right] \oplus\left(b\left(\mathcal{L}_{X^{1}} \omega^{2}-\mathcal{L}_{X^{2}} \omega^{1}\right)+c \mathrm{~d}\left\langle X^{2} \oplus \omega^{1}, X^{1} \oplus \omega^{2}\right\rangle_{-}\right)
\end{gathered}
$$

for (uniquely determined by $A$ ) real numbers $a, b, c$.

Roughly speaking, Corollary 2.4 says that if $m \geq 2$, then any $\mathcal{M} f_{m^{-}}$ natural skew-symmetric bilinear operator $A$ in the sense of Definition 2.1 coincides with the one given by Courant bracket $[-,-]^{C}$ up to three real constants.

Definition 2.5. A $\mathcal{M} f_{m}$-natural bilinear operator $A$ in the sense of Definition 2.1 satisfies the Leibniz rule if

$$
A\left(\rho_{1}, A\left(\rho_{2}, \rho_{3}\right)\right)=A\left(A\left(\rho_{1}, \rho_{2}\right), \rho_{3}\right)+A\left(\rho_{2}, A\left(\rho_{1}, \rho_{3}\right)\right)
$$

for all $\rho_{1}, \rho_{2}, \rho_{3} \in \mathcal{X}(M) \oplus \Omega^{1}(M)$ and all $m$-manifolds $M$.

Of course, in the case of skew-symmetric bilinear $A$ the Leibniz rule is equivalent to the Jacobi identity $\sum_{\operatorname{cycl}\left(\rho_{1}, \rho_{2}, \rho_{3}\right)} A\left(\rho_{1}, A\left(A\left(\rho_{2}, \rho_{3}\right)\right)=0\right.$. 
Example 2.6. The (not skew-symmetric) Courant bracket given by

$$
\begin{gathered}
{\left[X^{1} \oplus \omega^{1}, X^{2} \oplus \omega^{2}\right]_{0}} \\
:=\left[X^{1}, X^{2}\right] \oplus\left(\mathcal{L}_{X^{1}} \omega^{2}-i_{X^{2}} \mathrm{~d} \omega^{1}\right),
\end{gathered}
$$

where $X^{i} \oplus \omega^{i} \in \mathcal{X}(M) \oplus \Omega^{1}(M)$, satisfies the Leibniz rule, see [7,8].

The Courant bracket $[-,-]^{C}$ from Example 2.2 does not satisfy the Leibniz rule.

Theorem 2.7 [2]. If $m \geq 2$, any $\mathcal{M} f_{m}$-natural bilinear operator $A$ in the sense of Definition 2.1 satisfying the Leibniz rule is one of the following ones:

$$
\begin{aligned}
A^{\langle 1, a\rangle}\left(\rho^{1}, \rho^{2}\right) & =a\left[X^{1}, X^{2}\right] \oplus 0, \\
A^{\langle 2, a\rangle}\left(\rho^{1}, \rho^{2}\right) & =a\left[X^{1}, X^{2}\right] \oplus\left(a\left(\mathcal{L}_{X^{1}} \omega^{2}-\mathcal{L}_{X^{2}} \omega^{1}\right)\right), \\
A^{\langle 3, a\rangle}\left(\rho^{1}, \rho^{2}\right) & =a\left[X^{1}, X^{2}\right] \oplus a \mathcal{L}_{X^{1} \omega^{2},} \\
A^{\langle 4, a, 0\rangle}\left(\rho^{1}, \rho^{2}\right) & =a\left[X^{1}, X^{2}\right] \oplus\left(a\left(\mathcal{L}_{X^{1}} \omega^{2}-i_{X^{2}} \mathrm{~d} \omega^{1}\right)\right),
\end{aligned}
$$

where $a$ is an arbitrary real number, and where $\rho^{1}=X^{1} \oplus \omega^{1}$ and $\rho^{2}=$ $X^{2} \oplus \omega^{2}$.

Corollary 2.8. If $m \geq 2$, the Courant bracket $[-,-]_{0}$ from Example 2.6 for $m$-manifolds $M$ is the unique $\mathcal{M} f_{m}$-natural bilinear operator $A$ in the sense of Definition 2.1 satisfying the conditions:

(A1) $A\left(\rho_{1}, A\left(\rho_{2}, \rho_{3}\right)\right)=A\left(A\left(\rho_{1}, \rho_{2}\right), \rho_{3}\right)+A\left(\rho_{2}, A\left(\rho_{1}, \rho_{3}\right)\right)$,

(A2) $\pi A\left(\rho_{1}, \rho_{2}\right)=\left[\pi \rho_{1}, \pi \rho_{2}\right]$,

(A3) $A\left(\rho_{1}, \rho_{1}\right)=i_{0} \mathrm{~d}\left\langle\rho_{1}, \rho_{1}\right\rangle_{+}$,

for all $\rho_{1}, \rho_{2}, \rho_{3} \in \mathcal{X}(M) \oplus \Omega^{1}(M)$ and all m-manifolds $M$, where $\langle-,-\rangle_{+}$ is the pairing of Example 2.2, $\pi: T M \oplus T^{*} M \rightarrow T M$ is the fibred projection given by $\pi(v, \omega)=v$ and $i_{0}: T^{*} M \rightarrow T M \oplus T^{*} M$ is the fibred embedding $i_{0}(\omega)=(0, \omega)$.

Consequently, if $m \geq 2$, then a $\mathcal{M} f_{m}$-natural bilinear operator $A$ in the sense of Definition 2.1 satisfying the conditions (A1)-(A3) satisfies the conditions:

(A4) $\pi \rho_{1}\left\langle\rho_{2}, \rho_{3}\right\rangle_{+}=\left\langle A\left(\rho_{1}, \rho_{2}\right), \rho_{3}\right\rangle_{+}+\left\langle\rho_{2}, A\left(\rho_{1}, \rho_{3}\right\rangle_{+}\right.$,

(A5) $A\left(\rho_{1}, f \rho_{2}\right)=\pi \rho_{1}(f) \rho_{2}+f A\left(\rho_{1}, \rho_{2}\right)$

for all $\rho_{1}, \rho_{2} \in \mathcal{X}(M) \oplus \Omega^{1}(M)$, all $f \in \mathcal{C}^{\infty}(M)$ and all m-manifolds $M$ (i.e., putting $[[-,-]]:=A$ we get an exact Courant algebroid $E=(T M \oplus$ $\left.T^{*} M,[[-,-]],\langle-,-\rangle_{+}, \pi, i_{0}\right)$ in the sense of [8] for any $m$-manifold $\left.M\right)$.

Proof. By Theorem 2.7, the conditions $(A 1)$ and $(A 2)$ imply that $A=A^{\langle 1,1\rangle}$ or $A=A^{\langle 2,1\rangle}$ or $A=A^{\langle 3,1\rangle}$ or $A=A^{\langle 4,1,0\rangle}$. On the other hand if $\rho_{1}=X \oplus \omega$, then $i_{0} \mathrm{~d}\left\langle\rho_{1}, \rho_{1}\right\rangle_{+}=0 \oplus \mathrm{d} i_{X} \omega$ and $A^{\langle 1,1\rangle}\left(\rho_{1}, \rho_{1}\right)=0 \oplus 0$ and $A^{\langle 2,1\rangle}\left(\rho_{1}, \rho_{1}\right)=$ $0 \oplus 0$ and $A^{\langle 3,1\rangle}\left(\rho_{1}, \rho_{1}\right)=0 \oplus \mathcal{L}_{X} \omega$ and $A^{\langle 4,1,0\rangle}\left(\rho_{1}, \rho_{1}\right)=0 \oplus \mathrm{d} i_{X} \omega$. Then $A=A^{\langle 4,1,0\rangle}$.

Corollary 2.9. If $m \geq 2$, any $\mathcal{M} f_{m}$-natural Lie algebra brackets on $\mathcal{X}(M) \oplus$ $\Omega^{1}(M)$ [i.e., $\mathcal{M} f_{m}$-natural skew-symmetric bilinear operator satisfying the 
Jacobi identity (Leibniz rule) is the constant multiple of the one of the following two Lie algebra brackets:

$$
\begin{gathered}
{\left[\left[X^{1} \oplus \omega^{1}, X^{2} \oplus \omega^{2}\right]\right]_{1}=\left[X^{1}, X^{2}\right] \oplus 0,} \\
{\left[\left[X^{1} \oplus \omega^{1}, X^{2} \oplus \omega^{2}\right]\right]_{2}=\left[X^{1}, X^{2}\right] \oplus\left(\mathcal{L}_{X^{1}} \omega^{2}-\mathcal{L}_{X^{2}} \omega^{1}\right) .}
\end{gathered}
$$

At the end of this section we are going to describe completely all Lie algebroids $\left(T M \otimes T^{*} M,[[-,-]], a\right)$ which are invariant with respect to immersions $\left(\mathcal{M} f_{m}\right.$-maps). The concept of Lie algebroids can be found in the fundamental book [6].

Of course, the anchor $a: T M \oplus T^{*} M \rightarrow T M$ for all $m$-manifolds $M$ must be $\mathcal{M} f_{m}$-natural transformation [i.e., $T f \circ a=a \circ\left(T f \oplus T^{*} f\right)$ for any $\mathcal{M} f_{m}$-map $\left.f: M \rightarrow M^{1}\right]$ and fibre linear. By Corollary 2.9, $[[-,-]]=$ $\mu[[-,-]]_{1}$ or $[[-,-]]=\mu[[-,-]]_{2}$ for some $\mu \in \mathbf{R}$.

Lemma 2.10. Any $\mathcal{M} f_{m}$-natural transformation a: $T M \oplus T^{*} M \rightarrow T M$ which is fibre linear is the constant multiple of the fibre projection $\pi: T M \oplus$ $T^{*} M \rightarrow T M$.

Proof. Clearly, $a$ is determined by the values $<\eta, a_{x}(v, \omega)>\in \mathbf{R}$ for all $\omega, \eta \in T_{x}^{*} M, v \in T_{x} M, x \in M, M \in \operatorname{Obj}\left(\mathcal{M} f_{m}\right)$. By the standard chart arguments, we may assume $M=\mathbf{R}^{m}, x=0, \eta=\mathrm{d}_{0} x^{1}$. We can write $<\mathrm{d}_{0} x^{1}, a_{0}(v, \omega)>=\sum_{i} \alpha_{i} v^{i}+\sum_{j} \beta^{j} \omega_{j}$, where $v^{i}$ are the coordinates of $v$ and $\omega_{j}$ are the coordinates of $\omega$, and where $\alpha_{i}$ and $\beta^{j}$ are the real numbers determined by $a_{0}$. Then using the invariance of $a_{0}$ with respect to the maps $\left(\tau^{1} x^{1}, \ldots, \tau^{m} x^{m}\right)$ for $\tau^{1}>0, \ldots, \tau^{m}>0$ we deduce that $\alpha_{2}=\cdots=\alpha_{m}=0$ and $\beta_{1}=\cdots=\beta_{m}=0$. Then the vector space of all $a$ in question is at most 1-dimensional. Thus the dimension argument completes the proof.

So, $a=k \pi$ for some real number $k$. It must be $a\left(\left[\left[X^{1} \oplus 0, X^{2} \oplus 0\right]\right]\right)=$ $\left[a\left(X^{1} \oplus 0\right), a\left(X^{2} \oplus 0\right)\right]$ for any vector fields $X^{1}$ and $X^{2}$ on $M$. This gives the condition $k \mu\left[X^{1}, X^{2}\right]=k^{2}\left[X^{1}, X^{2}\right]$. Then $k \mu=k^{2}$, and then $(k=0$ and $\mu$ arbitrary) or $(k \neq 0$ and $\mu=k)$. Consider two cases:

1. $[[-,-]]=\mu[[-,-]]_{1}$. Let $\rho^{1}=X^{1} \oplus \omega^{1}$ and $\rho^{2}=X^{2} \oplus \omega^{2}$. It must be $\left[\left[\rho^{1}, f \rho^{2}\right]\right]=a\left(\rho^{1}\right)(f) \rho^{2}+f\left[\left[\rho^{1}, \rho^{2}\right]\right]$. Considering the $\Omega^{1}(M)$-parts of both sides of this equality we get $0=k X^{1}(f) \omega^{2}+0$ for any vector fields $X^{1}, X^{2}$ on $M$ any map $f: M \rightarrow \mathbf{R}$ and any $\omega^{1}, \omega^{2} \in \Omega^{1}(M)$. Then $k=0$. Then considering the $\mathcal{X}(M)$-parts we get $\mu\left[X^{1}, f X^{2}\right]=f \mu\left[X^{1}, X^{2}\right]$. Then $\mu X^{1}(f) X^{2}=0$ for all vector fields $X^{1}$ and $X^{2}$ on $M$ and all maps $f: M \rightarrow \mathbf{R}$, i.e., $\mu=0$.

2. $[[-,-]]=\mu[[-,-]]_{2}$. Let $\rho^{1}=0 \oplus \omega^{1}$ and $\rho^{2}=X^{2} \oplus 0$. It must be $\left[\left[\rho^{1}, f \rho^{2}\right]\right]=a\left(\rho^{1}\right)(f) \rho^{2}+f\left[\left[\rho^{1}, \rho^{2}\right]\right]$. Considering the $\Omega^{1}(M)$-parts of both sides of this equality we get $-\mu \mathcal{L}_{f X^{2}} \omega^{1}=-\mu f \mathcal{L}_{X^{2}} \omega^{1}$. Then $\mu=0$ or

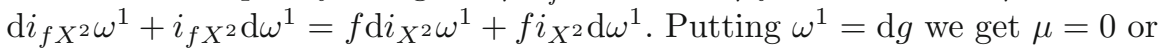
$\mathrm{d}\left(i_{f X^{2}} \mathrm{~d} g\right)=f \mathrm{~d} i_{X^{2}} \mathrm{~d} g$. Then $\mu=0$ or $\mathrm{d}\left(f X^{2} g\right)=f \mathrm{~d}\left(X^{2} g\right)$. Then $\mu=0$ or $X^{2}(g) \mathrm{d} f=0$ for any $X^{2}, g, f$ in question. Putting $X^{2}=\frac{\partial}{\partial x^{1}}$ and $f=g=x^{1}$ we get $\mu=0$ or $\mathrm{d} x^{1}=0$. Then $\mu=0$, and then $k=\mu=0$.

On the other hand one can directly show that $\left(T M \oplus T^{*} M, 0[[-,-]]_{1}, 0 \pi\right)$ is a Lie algebroid. Thus we have 
Proposition 2.11. If $m \geq 2,\left(T M \otimes T^{*} M, 0,0\right)$ is the only invariant with respect to $\mathcal{M} f_{m}$-maps Lie algebroid $(E M,[[-,-]], a)$ with $E M=T M \oplus$ $T^{*} M$.

\section{The Natural Operators Similar to the Twisted Courant Bracket}

Definition 3.1. A $\mathcal{M} f_{m}$-natural operator $A$ sending $p$-forms $H \in \Omega^{p}(M)$ on $m$-manifolds $M$ into bilinear operators

$$
A_{H}:\left(\mathcal{X}(M) \oplus \Omega^{1}(M)\right) \times\left(\mathcal{X}(M) \oplus \Omega^{1}(M)\right) \rightarrow \mathcal{X}(M) \oplus \Omega^{1}(M),
$$

is a $\mathcal{M} f_{m}$-invariant family of regular operators (functions)

$A: \Omega^{p}(M) \rightarrow \operatorname{Lin}_{2}\left(\left(\mathcal{X}(M) \oplus \Omega^{1}(M)\right) \times\left(\mathcal{X}(M) \oplus \Omega^{1}(M)\right), \mathcal{X}(M) \oplus \Omega^{1}(M)\right)$

for all $m$-manifolds $M$, where $\operatorname{Lin}_{2}(U \times V, W)$ denotes the vector space of all bilinear (over $\mathbf{R}$ ) functions $U \times V \rightarrow W$ for any real vector spaces $U, V, W$.

The $\mathcal{M} f_{m}$-invariance of $A$ means that if $H^{1} \in \Omega^{p}(M)$ and $H^{2} \in \Omega^{p}(\bar{M})$ are $\varphi$-related and $\left(X^{1} \oplus \omega^{1}, X^{2} \oplus \omega^{2}\right)$ and $\left(\bar{X}^{1} \oplus \bar{\omega}^{1}, \bar{X}^{2} \oplus \bar{\omega}^{2}\right)$ are $\varphi$-related by an $\mathcal{M} f_{m}$-map $\varphi: M \rightarrow \bar{M}$, then so are $A_{H^{1}}\left(X^{1} \oplus \omega^{1}, X^{2} \oplus \omega^{2}\right)$ and $A_{H^{2}}\left(\bar{X}^{1} \oplus \bar{\omega}^{1}, \bar{X}^{2} \oplus \bar{\omega}^{2}\right)$.

The regularity of $A$ means that it transforms smoothly parametrized families $\left(H_{t}, X_{t}^{1} \oplus \omega_{t}^{1}, X_{t}^{2} \oplus \omega_{t}^{2}\right)$ into smoothly parametrized families $A_{H_{t}}\left(X_{t}^{1} \oplus\right.$ $\left.\omega_{t}^{1}, X_{t}^{2} \oplus \omega_{t}^{2}\right)$.

Example 3.2. The most important example of $\mathcal{M} f_{m}$-natural operator in the sense of Definition 3.1 for $p=3$ is given by the $H$-twisted Courant bracket

$$
\left[X^{1} \oplus \omega^{1}, X^{2} \oplus \omega^{2}\right]_{H}:=\left[X^{1}, X^{2}\right] \oplus\left(\mathcal{L}_{X^{1}} \omega^{2}-i_{X^{2}} \mathrm{~d} \omega^{1}+i_{X^{1}} i_{X^{2}} H\right)
$$

for all 3-forms $H \in \Omega^{3}(M)$ and all $m$-manifolds $M$. We call this $\mathcal{M} f_{m}$-natural operator the twisted Courant bracket $\mathcal{M} f_{m}$-natural operator.

Example 3.3. The operator given by $[-,-]_{\mathrm{d} H}$ for all $H \in \Omega^{2}(M)$ and all $m$-manifolds $M$ is a $\mathcal{M} f_{m}$-natural operator in the sense of Definition 3.1 for $p=2$.

The main result of this section is the following

Theorem 3.4. Assume $m \geq 3$. Then we have:

1. Any $\mathcal{M} f_{m}$-natural operator $A$ in the sense of Definition 3.1 for $p=2$ such that $A_{H}=A_{H+\mathrm{d} H^{1}}$ for any $H \in \Omega^{2}(M)$ and any $H^{1} \in \Omega^{1}(M)$ is of the form

$$
\begin{gathered}
A_{H}\left(\rho^{1}, \rho^{2}\right)=a\left[X^{1}, X^{2}\right] \\
\oplus\left(b_{1} \mathcal{L}_{X^{2}} \omega^{1}+b_{2} \mathcal{L}_{X^{1}} \omega^{2}+b_{3} \mathrm{~d}\left\langle\rho^{1}, \rho^{2}\right\rangle_{+}+b_{4} \mathrm{~d}\left\langle\rho^{1}, \rho^{2}\right\rangle_{-}+c i_{X^{1}} i_{X^{2}} \mathrm{~d} H\right),
\end{gathered}
$$

for (uniquely determined by $A$ ) reals $a, b_{1}, \ldots, c$, where 2 -forms $H \in$ $\Omega^{2}(M)$, pairs $\rho^{i}=X^{i} \oplus \omega^{i} \in \mathcal{X}(M) \oplus \Omega^{1}(M)$ for $i=1,2$ and $m$ manifolds $M$ are arbitrary. 
2. Any $\mathcal{M} f_{m}$-natural operator (not necessarily satisfying $A_{H}=A_{H+\mathrm{d} H^{1}}$ ) in the sense of Definition 3.1 for $p=3$ is of the form

$$
\begin{gathered}
A_{H}\left(\rho^{1}, \rho^{2}\right)=a\left[X^{1}, X^{2}\right] \\
\oplus\left(b_{1} \mathcal{L}_{X^{2}} \omega^{1}+b_{2} \mathcal{L}_{X^{1}} \omega^{2}+b_{3} \mathrm{~d}\left\langle\rho^{1}, \rho^{2}\right\rangle_{+}+b_{4} \mathrm{~d}\left\langle\rho^{1}, \rho^{2}\right\rangle_{-}+c i_{X^{1}} i_{X^{2}} H\right),
\end{gathered}
$$

for (uniquely determined by $A$ ) reals a, $b_{1}, \ldots, c$, where 3 -forms $H \in$ $\Omega^{3}(M)$, pairs $\rho^{i}=X^{i} \oplus \omega^{i} \in \mathcal{X}(M) \oplus \Omega^{1}(M)$ for $i=1,2$ and $m$ manifolds $M$ are arbitrary.

3. If $p \geq 4$, any $\mathcal{M} f_{m}$-natural operator (not necessarily satisfying $A_{H}=$ $\left.A_{H+\mathrm{d} H^{1}}\right)$ in the sense of Definition 3.1 is of the form

$$
A_{H}\left(\rho^{1}, \rho^{2}\right)=a\left[X^{1}, X^{2}\right] \oplus\left(b_{1} \mathcal{L}_{X^{2}} \omega^{1}+b_{2} \mathcal{L}_{X^{1}} \omega^{2}+b_{3} \mathrm{~d}\left\langle\rho^{1}, \rho^{2}\right\rangle_{+}+b_{4} \mathrm{~d}\left\langle\rho^{1}, \rho^{2}\right\rangle_{-}\right)
$$

for (uniquely determined by $A$ ) reals $a, b_{1}, \ldots, b_{4}$, where $p$-forms $H \in$ $\Omega^{p}(M)$, pairs $\rho^{i}=X^{i} \oplus \omega^{i} \in \mathcal{X}(M) \oplus \Omega^{1}(M)$ for $i=1,2$ and $m$ manifolds $M$ are arbitrary.

Proof. Clearly, $A_{0}$, where 0 is the zero $p$-form, can be treated as the bilinear operator in the sense of Definition 2.1. Then $A_{0}$ is described in Theorem 2.3. So we can replace $A$ by $A-A_{0}$. In other words, we have assumption $A_{0}=0$.

By the invariance, $A$ is determined by the values $A_{H}\left(X^{1} \oplus \omega^{1}, X^{2} \oplus\right.$ $\left.\omega^{2}\right)_{\left.\right|_{0}}$ for all $H \in \Omega^{p}\left(\mathbf{R}^{m}\right), X^{i} \oplus \omega^{i} \in \mathcal{X}\left(\mathbf{R}^{m}\right) \oplus \Omega^{1}\left(\mathbf{R}^{m}\right)$. Put

$$
\begin{aligned}
& A_{H}\left(X^{1} \oplus \omega^{1}, X^{2} \oplus \omega^{2}\right)_{\mid 0} \\
& \quad=\left(A_{H}^{1}\left(X^{1} \oplus \omega^{1}, X^{2} \oplus \omega^{2}\right)_{\mid 0}, A_{H}^{2}\left(X^{1} \oplus \omega^{1}, X^{2} \oplus \omega^{2}\right)_{\mid 0}\right),
\end{aligned}
$$

where $A_{H}^{1}(\ldots)_{\mid 0} \in T_{0} \mathbf{R}^{m}$ and $A_{H}^{2}(\ldots)_{\mid 0} \in T^{*} \mathbf{R}^{m}$. Then $A$ is determined by

$$
\left\langle A_{H}^{1}\left(X^{1} \oplus \omega^{1}, X^{2} \oplus \omega^{2}\right)_{\left.\right|_{0}}, \eta\right\rangle \in \mathbf{R} \text { and }\left\langle A_{H}^{2}\left(X^{1} \oplus \omega^{1}, X^{2} \oplus \omega^{2}\right)_{\mid 0}, \mu\right\rangle \in \mathbf{R}
$$

for all $H \in \Omega^{p}\left(\mathbf{R}^{m}\right), X^{i} \oplus \omega^{i} \in \mathcal{X}\left(\mathbf{R}^{m}\right) \oplus \Omega^{1}\left(\mathbf{R}^{m}\right), \eta \in T_{0}^{*} \mathbf{R}^{m}, \mu \in T_{0} \mathbf{R}^{m}$, $i=1,2$.

By the non-linear Peetre theorem, see [5], $A$ is of finite order. It means that there is a finite number $r$ such that from $\left(j_{x}^{r} H=j_{x}^{r} \bar{H}, j_{x}^{r}\left(\rho^{i}\right)=j_{x}^{r}\left(\bar{\rho}^{i}\right)\right.$, $i=1,2)$ it follows $A_{H}\left(\rho^{1}, \rho^{2}\right)_{\mid x}=A_{\bar{H}}\left(\bar{\rho}^{1}, \bar{\rho}^{2}\right)_{\mid x}$. So, we may assume that $H, X^{1}, X^{2}, \omega^{1}, \omega^{2}$ are polynomials of degree not more than $r$.

Using the invariance of $A$ with respect to the homotheties and the bilinearity of $A_{H}$ (for given $H$ ) we obtain homogeneity condition

$$
\begin{gathered}
\left\langleA _ { ( \frac { 1 } { t } \mathrm { id } ) _ { * } H } ^ { 1 } \left( t\left(\frac{1}{t} \mathrm{id}\right)_{*} X^{1} \oplus t\left(\frac{1}{t} \mathrm{id}\right)_{*} \omega^{1}, t\left(\frac{1}{t} \mathrm{id}\right)_{*} X^{2}\right.\right. \\
\left.\left.\oplus t\left(\frac{1}{t} \mathrm{id}\right)_{*} \omega^{2}\right)_{\left.\right|_{0}}, \eta\right\rangle \\
=t\left\langle A_{H}^{1}\left(X^{1} \oplus \omega^{1}, X^{2} \oplus \omega^{2}\right)_{\left.\right|_{0}}, \eta\right\rangle .
\end{gathered}
$$

Then, by the homogeneous function theorem, since $A$ is of finite order and regular and $A_{0}=0$ and $p \geq 2$, we have $\left\langle A_{H}^{1}\left(X^{1} \oplus \omega^{1}, X^{2} \oplus \omega^{2}\right)_{\left.\right|_{0}}, \eta\right\rangle=0$. 
Using the same arguments we get homogeneity condition

$$
\begin{gathered}
\left\langleA _ { ( \frac { 1 } { t } \mathrm { id } ) _ { * } H } ^ { 2 } \left( t\left(\frac{1}{t} \mathrm{id}\right)_{*} X^{1} \oplus t\left(\frac{1}{t} \mathrm{id}\right)_{*} \omega^{1}, t\left(\frac{1}{t} \mathrm{id}\right)_{*} X^{2}\right.\right. \\
\left.\left.\oplus t\left(\frac{1}{t} \mathrm{id}\right)_{*} \omega^{2}\right)_{\left.\right|_{0}}, \mu\right\rangle \\
=t^{3}\left\langle A_{H}^{2}\left(X^{1} \oplus \omega^{1}, X^{2} \oplus \omega^{2}\right)_{\left.\right|_{0}}, \mu\right\rangle .
\end{gathered}
$$

Then, if $p=2$, by the homogeneous function theorem and the bilinearity of $A_{H}$ and the assumptions $A_{0}=0$ and $A_{H}=A_{H+\mathrm{d} H^{1}}$, the value $\left\langle A_{H}^{2}\left(X^{1} \oplus \omega^{1}, X^{2} \oplus \omega^{2}\right)_{\mid 0}, \mu\right\rangle$ depends quadrilinearly on $X_{\mid 0}^{1}, X_{\mid 0}^{2}$, $j_{0}^{1}\left(H-H_{\mid 0}\right)$ and $\mu$, only. By $m \geq 3$ and the regularity of $A$, we may assume that $X_{\mid 0}^{1}, X_{\mid 0}^{2}$ and $\mu$ are linearly independent. Then by the invariance we may assume $X_{\mid 0}^{1}=\partial_{1 \mid 0}, X_{\mid 0}^{2}=\partial_{2 \mid 0}$ and $\mu=\partial_{3 \mid 0}$. Then $A$ is determined by the values $\left\langle A_{x^{i_{1} \mathrm{~d} x^{i_{2}} \wedge \mathrm{d} x^{i_{3}}}}^{2}\left(\partial_{1} \oplus 0, \partial_{2} \oplus 0\right), \partial_{3 \mid 0}\right\rangle$ for all $i_{1}=1, \ldots, m$ and $i_{2}, i_{3}$ with $1 \leq i_{2}<i_{3} \leq m$. Then using the invariance of $A$ with respect to $\tau$ id for $\tau^{i}>0$ we deduce that only $v:=\left\langle A_{x^{1} \mathrm{~d} x^{2} \wedge \mathrm{d} x^{3}}^{2}\left(\partial_{1} \oplus 0, \partial_{2} \oplus 0\right), \partial_{3 \mid 0}\right\rangle$, $w:=\left\langle A_{x^{2} \mathrm{~d} x^{1} \wedge \mathrm{d} x^{3}}^{2}\left(\partial_{1} \oplus 0, \partial_{2} \oplus 0\right), \partial_{3 \mid 0}\right\rangle, z:=\left\langle A_{x^{3} \mathrm{~d} x^{1} \wedge \mathrm{d} x^{2}}^{2}\left(\partial_{1} \oplus 0, \partial_{2} \oplus 0\right), \partial_{3 \mid 0}\right\rangle$ may be not-zero. But $x^{1} \mathrm{~d} x^{2} \wedge \mathrm{d} x^{3}=-x^{2} \mathrm{~d} x^{1} \wedge \mathrm{d} x^{3}+\mathrm{d}(\ldots)$. So, $v=-w$. Similarly, $v=-z$. Therefore the vector space of all $A$ in question with $A_{0}=0$ and $A_{H}=A_{H+\mathrm{d} H^{1}}$ is at most one-dimensional. The part (1) of the theorem is complete. If $p=3$, then (by almost the same arguments as for $p=2) A$ is determined by the values $\left\langle A_{\mathrm{d} x^{i_{1}} \wedge \mathrm{d} x^{i_{2}} \wedge \mathrm{d} x^{i_{3}}}^{2}\left(\partial_{1} \oplus 0, \partial_{2} \oplus 0\right), \partial_{3 \mid 0}\right\rangle \in$ $\mathbf{R}$ for all $i_{1}, i_{2}, i_{3}$ with $1 \leq i_{1}<i_{2}<i_{3} \leq m$. Then using the invariance with respect to $\left(\tau^{1} x^{1}, \ldots \tau^{m} x^{m}\right)$ for $\tau^{i}>0$ we deduce that only the value $\left\langle A_{\mathrm{d} x^{1} \wedge \mathrm{d} x^{2} \wedge \mathrm{d} x^{3}}^{2}\left(\partial_{1} \oplus 0, \partial_{2} \oplus 0\right), \partial_{3 \mid 0}\right\rangle \in \mathbb{R}$ may be not-zero. Therefore the vector space of all $A$ in question with $A_{0}=0$ is one-dimensional (generated by the natural operator $\left.0 \oplus i_{X^{1}} i_{X^{2}} H\right)$.

If $p \geq 4$, then (similarly as for $p=2)<A_{H}^{2}\left(X^{1} \oplus \omega^{1}, X^{2} \oplus \omega^{2}\right)_{\mid 0}, \mu>=0$.

Theorem 3.4 is complete.

Corollary 3.5. If $m \geq 3$, any $\mathcal{M} f_{m}$-natural operator $A$ in the sense of Definition 3.1 for $p=3$ such that $A_{H}$ is skew-symmetric for any $H \in \Omega^{3}(M)$ and any $m$-manifold $M$ is of the form

$$
\begin{aligned}
& A_{H}\left(X^{1} \oplus \omega^{1}, X^{2} \oplus \omega^{2}\right)=a\left[X^{1}, X^{2}\right] \\
& \quad \oplus\left(b\left(\mathcal{L}_{X^{1}} \omega^{2}-\mathcal{L}_{X^{2}} \omega^{1}\right)+c \mathrm{~d}\left\langle X^{2} \oplus \omega^{1}, X^{1} \oplus \omega^{2}\right\rangle_{-}+e i_{X^{1}} i_{X^{2}} H\right)
\end{aligned}
$$

for (uniquely determined by $A$ ) real numbers $a, b, c, e$.

Roughly speaking, Corollary 3.5 says that any $\mathcal{M} f_{m}$-natural operator $A$ in the sense of Definition 3.1 such that $A_{H}$ is skew-symmetric for any $H \in$ $\Omega^{3}(M)$ and any $m$-manifold $M$ coincides with the "skew-symmetrization" of the twisted Courant bracket $\mathcal{M} f_{m}$-natural operator up to four real constants $a, b, c, e$.

Corollary 3.6. If $m \geq 3$, then the twisted Courant bracket $\mathcal{M} f_{m}$-natural operator from Example 3.2 is the unique $\mathcal{M} f_{m}$-natural operator $A$ in the sense of Definition 3.1 for $p=3$ satisfying the following properties: 
(B1) $A_{0}\left(\rho_{1}, \rho_{2}\right)=\left[\rho_{1}, \rho_{2}\right]_{0}$,

(B2) $A_{H}(X \oplus 0, Y \oplus 0)=[X, Y] \oplus i_{X} i_{Y} H$

for all closed $H \in \Omega_{c l}^{3}(M)$, all $\rho_{1}, \rho_{2}, X \oplus 0, Y \oplus 0 \in \mathcal{X}(M) \oplus \Omega^{1}(M)$ and all $m$-manifolds $M$, where $[-,-]_{0}$ is the $\mathcal{M} f_{m}$-natural bilinear operator given by the (not skew-symmetric) Courant bracket as in Example 2.6.

Proof. Clearly, the twisted Courant bracket $\mathcal{M} f_{m}$-natural operator satisfies $(B 1)$ and (B2). Consider $A$ in question satisfying (B1) and (B2). Then by Theorem 3.4, there exist uniquely determined reals $a, b_{1}, \ldots, c$ such that for all $H \in \Omega^{3}(M)$ and $m$-manifolds $M$

$$
\begin{gathered}
A_{H}\left(\rho^{1}, \rho^{2}\right)=a\left[X^{1}, X^{2}\right] \\
\oplus\left(b_{1} \mathcal{L}_{X^{2}} \omega^{1}+b_{2} \mathcal{L}_{X^{1}} \omega^{2}+b_{3} \mathrm{~d}\left\langle\rho^{1}, \rho^{2}\right\rangle_{+}+b_{4} \mathrm{~d}\left\langle\rho^{1}, \rho^{2}\right\rangle_{-}+c i_{X^{1}} i_{X^{2}} H\right),
\end{gathered}
$$

where $\rho^{i}=X^{i} \oplus \omega^{i} \in \mathcal{X}(M) \oplus \Omega^{1}(M)$ are arbitrary. Putting $\omega^{1}=\omega^{2}=0$ we get $A_{H}\left(\rho^{1}, \rho^{2}\right)=a\left[X^{1}, X^{2}\right] \oplus c i_{X^{1}} i_{X^{2}} H$. Then condition $(B 2)$ implies $c=1$. Putting $H=0$ we get

$$
A_{0}\left(\rho^{1}, \rho^{2}\right)=a\left[X^{1}, X^{2}\right] \oplus\left(b_{1} \mathcal{L}_{X^{2}} \omega^{1}+b_{2} \mathcal{L}_{X^{1}} \omega^{2}+b_{3} \mathrm{~d}\left\langle\rho^{1}, \rho^{2}\right\rangle_{+}+b_{4} \mathrm{~d}\left\langle\rho^{1}, \rho^{2}\right\rangle_{-}\right)
$$

for all $\rho^{i}=X^{i} \oplus \omega^{i} \in \mathcal{X}(M) \oplus \Omega^{1}(M)$ and all $m$-manifolds $M$. But $A_{0}$ is a $\mathcal{M} f_{m}$-natural bilinear operator in the sense of Definition 2.1. Then $a, b_{1}, b_{2}, b_{3}, b_{4}$ are uniquely determined because of Theorem 2.3. Then $a$, $b_{1}, \ldots, c$ are uniquely determined. So, $A$ is uniquely determined by conditions $(B 1)$ and $(B 2)$.

\section{The Natural Operators Similar to the Twisted Courant Bracket and Defined for Closed $p$-Forms Only}

In the previous section, we considered $\mathcal{M} f_{m}$-natural operators $A$ which are defined for all $p$-forms $H$. In this section, we observe what happens if $A$ are defined for closed $p$-forms $H$, only. We start with the following

Definition 4.1. A $\mathcal{M} f_{m}$-natural operator $A$ sending closed $p$-forms $H \in \Omega_{c l}^{p}(M)$ on $m$-manifolds $M$ into bilinear operators

$$
A_{H}:\left(\mathcal{X}(M) \oplus \Omega^{1}(M)\right) \times\left(\mathcal{X}(M) \oplus \Omega^{1}(M)\right) \rightarrow \mathcal{X}(M) \oplus \Omega^{1}(M),
$$

is a $\mathcal{M} f_{m}$-invariant family of regular operators (functions)

$A: \Omega_{c l}^{p}(M) \rightarrow \operatorname{Lin}_{2}\left(\left(\mathcal{X}(M) \oplus \Omega^{1}(M)\right) \times\left(\mathcal{X}(M) \oplus \Omega^{1}(M)\right), \mathcal{X}(M) \oplus \Omega^{1}(M)\right)$, for all $m$-manifolds $M$.

We have the following corollary of Theorem 3.4.

Corollary 4.2. Assume $m \geq 3$. Then we have:

1. If $p=3$, any $\mathcal{M} f_{m}$-natural operator in the sense of Definition 4.1 is of the form

$$
\begin{gathered}
A_{H}\left(\rho^{1}, \rho^{2}\right)=a\left[X^{1}, X^{2}\right] \\
\oplus\left(b_{1} \mathcal{L}_{X^{2}} \omega^{1}+b_{2} \mathcal{L}_{X^{1}} \omega^{2}+b_{3} \mathrm{~d}\left\langle\rho^{1}, \rho^{2}\right\rangle_{+}+b_{4} \mathrm{~d}\left\langle\rho^{1}, \rho^{2}\right\rangle_{-}+c i_{X^{1}} i_{X^{2}} H\right),
\end{gathered}
$$


for uniquely determined by $A$ reals $a, b_{1}, \ldots, c$, where closed 3 -forms $H \in$ $\Omega_{c l}^{3}(M)$, pairs $\rho^{i}=X^{i} \oplus \omega^{i} \in \mathcal{X}(M) \oplus \Omega^{1}(M)$ for $i=1,2$ and $m$ manifolds $M$ are arbitrary.

2. If $p \geq 4$, any $\mathcal{M} f_{m}$-natural operator in the sense of Definition 4.1 is of the form

$$
\begin{gathered}
A_{H}\left(\rho^{1}, \rho^{2}\right)=a\left[X^{1}, X^{2}\right] \\
\oplus\left(b_{1} \mathcal{L}_{X^{2}} \omega^{1}+b_{2} \mathcal{L}_{X^{1}} \omega^{2}+b_{3} \mathrm{~d}\left\langle\rho^{1}, \rho^{2}\right\rangle_{+}+b_{4} \mathrm{~d}\left\langle\rho^{1}, \rho^{2}\right\rangle_{-}\right)
\end{gathered}
$$

for uniquely determined by $A$ reals $a, b_{1}, \ldots, b_{4}$, where closed $p$-forms $H \in \Omega_{c l}^{p}(M)$, pairs $\rho^{i}=X^{i} \oplus \omega^{i}$ for $i=1,2$ and $m$-manifolds $M$ are arbitrary.

Proof. Let $A$ be a $\mathcal{M} f_{m}$-natural operator in the sense of Definition 4.1 for $p$. Define a $\mathcal{M} f_{m}$-natural operator $A^{1}$ in the sense of Definition 3.1 for $p-1$ by $A_{\tilde{H}}^{1}=A_{\mathrm{d} \tilde{H}}$. Then $A_{\tilde{H}+\mathrm{d} H_{1}}^{1}=A_{\tilde{H}}^{1}$ for any $\tilde{H} \in \Omega^{p-1}(M)$ and $H_{1} \in \Omega^{p-2}(M)$.

If $p=3$, then by Theorem $3.4, A^{1}$ is of the form

$$
\begin{gathered}
A_{\tilde{H}}^{1}\left(\rho^{1}, \rho^{2}\right)=a\left[X^{1}, X^{2}\right] \\
\oplus\left(b_{1} \mathcal{L}_{X^{2}} \omega^{1}+b_{2} \mathcal{L}_{X^{1}} \omega^{2}+b_{3} \mathrm{~d}\left\langle\rho^{1}, \rho^{2}\right\rangle_{+}+b_{4} \mathrm{~d}\left\langle\rho^{1}, \rho^{2}\right\rangle_{-}+c i_{X^{1}} i_{X^{2}} \mathrm{~d} \tilde{H}\right)
\end{gathered}
$$

for uniquely determined reals $a, b_{1}, \ldots, c$ and all $\tilde{H} \in \Omega^{2}(M)$, where $\rho^{i}=$ $X^{i} \oplus \omega^{i}$ for $i=1,2$. Then

$$
\begin{gathered}
A_{H}\left(\rho^{1}, \rho^{2}\right)=a\left[X^{1}, X^{2}\right] \\
\oplus\left(b_{1} \mathcal{L}_{X^{2}} \omega^{1}+b_{2} \mathcal{L}_{X^{1}} \omega^{2}+b_{3} \mathrm{~d}\left\langle\rho^{1}, \rho^{2}\right\rangle_{+}+b_{4} \mathrm{~d}\left\langle\rho^{1}, \rho^{2}\right\rangle_{-}+c i_{X^{1}} i_{X^{2}} H\right)
\end{gathered}
$$

for all exact 3 -forms $H$, where $\rho^{i}=X^{i} \oplus \omega^{i}$ for $i=1,2$. But by the locality of $A$ and the Poincare lemma we may replace the phrase "all exact 3 -forms" by "all closed 3 -forms".

If $p \geq 4$, then by Theorem $3.4, A^{1}$ is of the form

$$
\begin{gathered}
A_{\tilde{H}}^{1}\left(\rho^{1}, \rho^{2}\right)=a\left[X^{1}, X^{2}\right] \\
\oplus\left(b_{1} \mathcal{L}_{X^{2}} \omega^{1}+b_{2} \mathcal{L}_{X^{1}} \omega^{2}+b_{3} \mathrm{~d}\left\langle\rho^{1}, \rho^{2}\right\rangle_{+}+b_{4} \mathrm{~d}\left\langle\rho^{1}, \rho^{2}\right\rangle_{-}+c i_{X^{1}} i_{X^{2}} \mathrm{~d} \tilde{H}\right)
\end{gathered}
$$

for uniquely determined reals $a, b_{1}, \ldots, c$ (with arbitrary $c$ if $p=4$ and with $c=0$ if $p \geq 5$ ) and all $\tilde{H} \in \Omega^{p-1}(M)$, where $\rho^{i}=X^{i} \oplus \omega^{i}$ for $i=1,2$. The condition $\bar{A}_{\tilde{H}}^{1}=A_{\tilde{H}+\mathrm{d} H_{1}}^{1}$ implies $c i_{X^{1}} i_{X^{2}} \mathrm{~d} H_{1}=0$ for any $H_{1} \in \Omega^{p-2}(M)$. If $p=4$, putting $X^{1}=\partial_{1}, X^{2}=\partial_{2}$ and $H_{1}=x^{1} \mathrm{~d} x^{2} \wedge \mathrm{d} x^{3}$, we get $c\left(-\mathrm{d} x^{3}\right)=0$, i.e., $c=0$. If $p \geq 5$, then $c=0$, see above. Next, we proceed similarly as in the case $p=3$.

The above corollary and Theorem 3.4 imply

Theorem 4.3. If $m \geq 3$ and $p \geq 3$ then any $\mathcal{M} f_{m}$-natural operator in the sense of Definition 4.1 can be extended uniquely to a $\mathcal{M} f_{m}$-natural operator in the sense of Definition 3.1.

Roughly speaking, if $m \geq 3$ and $p \geq 3$, then any $\mathcal{M} f_{m}$-natural operator in the sense of Definition 4.1 can be treated as the $\mathcal{M} f_{m}$-natural operator in the sense of Definition 3.1, and vice-versa. 


\section{The Natural Operators Similar to the Twisted Courant Bracket and Satisfying the Leibniz Rule for Closed 3-Forms}

Definition 5.1. A $\mathcal{M} f_{m}$-natural operator $A$ in the sense of Definition 3.1 (or equivalently in the sense of Definition 4.1) satisfies the Leibniz rule for closed $p$-forms if

$$
A_{H}\left(\rho_{1}, A_{H}\left(\rho_{2}, \rho_{3}\right)\right)=A_{H}\left(A_{H}\left(\rho_{1}, \rho_{2}\right), \rho_{3}\right)+A_{H}\left(\rho_{2}, A_{H}\left(\rho_{1}, \rho_{3}\right)\right)
$$

for all $\rho_{1}, \rho_{2}, \rho_{3} \in \mathcal{X}(M) \oplus \Omega^{1}(M)$, all closed $p$-forms $H \in \Omega_{c l}^{p}(M)$ and all $m$-manifolds $M$.

Example 5.2. The twisted Courant bracket $\mathcal{M} f_{m}$-natural operator presented in Example 3.2 satisfies the Leibniz rule for closed 3 -forms, see [3,8].

Theorem 5.3. If $m \geq 3$, any $\mathcal{M} f_{m}$-natural operator $A$ in the sense of Definition 3.1 (or equivalently of Definition 4.1) for $p=3$ satisfying the Leibniz rule for closed 3 -forms is one of the $\mathcal{M} f_{m}$-natural operators:

$$
\begin{array}{rlrl}
A_{H}^{\langle 1, a\rangle}\left(\rho_{1}, \rho_{2}\right) & = & a\left[X^{1}, X^{2}\right] \oplus 0, \\
A_{H}^{\langle 2, a\rangle}\left(\rho^{1}, \rho^{2}\right) & = & a\left[X^{1}, X^{2}\right] \oplus\left(a\left(\mathcal{L}_{X^{1}} \omega^{2}-\mathcal{L}_{X^{2}} \omega^{1}\right)\right), \\
A_{H}^{\langle 3, a\rangle}\left(\rho^{1}, \rho^{2}\right) & = & a\left[X^{1}, X^{2}\right] \oplus\left(a \mathcal{L}_{X^{1}} \omega^{2}\right), \\
A_{H}^{\langle 4, a, e\rangle}\left(\rho^{1}, \rho^{2}\right) & =a\left[X^{1}, X^{2}\right] \oplus\left(a\left(\mathcal{L}_{X^{1}} \omega^{2}-i_{X^{2}} \mathrm{~d} \omega^{1}\right)+e i_{X^{1}} i_{X^{2}} H\right),
\end{array}
$$

where $\rho^{1}=X^{1} \oplus \omega^{1}$ and $\rho^{2}=X^{2} \oplus \omega^{2}$, and a and $e$ are arbitrary real numbers.

Proof. Let $A$ be a $\mathcal{M} f_{m}$-natural operator in the sense of Definition 3.1 for $p=3$ such that $A_{H}$ satisfies the Leibniz rule for any closed $H \in \Omega_{c l}^{3}(M)$. By Theorem 3.4, $A$ is of the form

$$
\begin{gathered}
A_{H}\left(X^{1} \oplus \omega^{1}, X^{2} \oplus \omega^{2}\right)=a\left[X^{1}, X^{2}\right] \\
\oplus\left(b_{1} \mathcal{L}_{X^{2} \omega^{1}}+b_{2} \mathcal{L}_{X^{1}} \omega^{2}+c_{1} \mathrm{~d} i_{X^{2}} \omega^{1}+c_{2} \mathrm{~d} i_{X^{1}} \omega^{2}+e i_{X^{1}} i_{X^{2}} H\right),
\end{gathered}
$$

for (uniquely determined by $A$ ) real numbers $a, b_{1}, b_{2}, c_{1}, c_{2}, e$. Then for any $X^{1}, X^{2}, X^{3} \in \mathcal{X}(M)$ and $\omega^{1}, \omega^{2}, \omega^{3} \in \Omega^{1}(M)$ we have

$$
\begin{aligned}
& A_{H}\left(X^{1} \oplus \omega^{1}, A_{H}\left(X^{2} \oplus \omega^{2}, X^{3} \oplus \omega^{3}\right)\right)=a^{2}\left[X^{1},\left[X^{2}, X^{3}\right]\right] \oplus \Omega, \\
& A_{H}\left(A_{H}\left(X^{1} \oplus \omega^{1}, X^{2} \oplus \omega^{2}\right), X^{3} \oplus \omega^{3}\right)=a^{2}\left[\left[X^{1}, X^{2}\right], X^{3}\right] \oplus \Theta, \\
& A_{H}\left(X^{2} \oplus \omega^{2}, A_{H}\left(X^{1} \oplus \omega^{1}, X^{3} \oplus \omega^{3}\right)\right)=a^{2}\left[X^{2},\left[X^{1}, X^{3}\right]\right] \oplus \mathcal{T},
\end{aligned}
$$


where

$$
\begin{aligned}
& \Omega=b_{1} \mathcal{L}_{a\left[X^{2}, X^{3}\right]} \omega^{1}+c_{1} \mathrm{~d} i_{a\left[X^{2}, X^{3}\right]} \omega^{1}+e i_{X^{1}} i_{a\left[X^{2}, X^{3}\right]} H \\
& +b_{2} \mathcal{L}_{X^{1}}\left(b_{1} \mathcal{L}_{X^{3}} \omega^{2}+b_{2} \mathcal{L}_{X^{2}} \omega^{3}+c_{1} \mathrm{~d} i_{X^{3}} \omega^{2}+c_{2} \mathrm{~d} i_{X^{2}} \omega^{3}+e i_{X^{2}} i_{X^{3}} H\right) \\
& +c_{2} \mathrm{~d} i_{X^{1}}\left(b_{1} \mathcal{L}_{X^{3}} \omega^{2}+b_{2} \mathcal{L}_{X^{2}} \omega^{3}+c_{1} \mathrm{~d} i_{X^{3}} \omega^{2}+c_{2} \mathrm{~d} i_{X^{2}} \omega^{3}+e i_{X^{2}} i_{X^{3}} H\right), \\
& \Theta=b_{2} \mathcal{L}_{a\left[X^{1}, X^{2}\right]} \omega^{3}+c_{2} \mathrm{~d} i_{a\left[X^{1}, X^{2}\right]} \omega^{3}+e i_{a\left[X^{1}, X^{2}\right]} i_{X^{3}} H \\
& +b_{1} \mathcal{L}_{X^{3}}\left(b_{1} \mathcal{L}_{X^{2}} \omega^{1}+b_{2} \mathcal{L}_{X^{1}} \omega^{2}+c_{1} \mathrm{~d} i_{X^{2}} \omega^{1}+c_{2} \mathrm{~d} i_{X^{1}} \omega^{2}+e_{X^{1}} i_{X^{2}} H\right) \\
& +c_{1} \mathrm{~d} i_{X^{3}}\left(b_{1} \mathcal{L}_{X^{2}} \omega^{1}+b_{2} \mathcal{L}_{X^{1}} \omega^{2}+c_{1} \mathrm{~d} i_{X^{2}} \omega^{1}+c_{2} \mathrm{~d} i_{X^{1}} \omega^{2}+e i_{X^{1}} i_{X^{2}} H\right), \\
& \mathcal{T}=b_{1} \mathcal{L}_{a\left[X^{1}, X^{3}\right]} \omega^{2}+c_{1} \mathrm{~d} i_{a\left[X^{1}, X^{3}\right]} \omega^{2}+e i_{X^{2}} i_{a\left[X^{1}, X^{3}\right]} H \\
& +b_{2} \mathcal{L}_{X^{2}}\left(b_{1} \mathcal{L}_{X^{3}} \omega^{1}+b_{2} \mathcal{L}_{X^{1}} \omega^{3}+c_{1} \mathrm{~d} i_{X^{3} \omega^{1}}+c_{2} \mathrm{~d} i_{X^{1}} \omega^{3}+e i_{X^{1}} i_{X^{3}} H\right) \\
& +c_{2} \mathrm{~d} i_{X^{2}}\left(b_{1} \mathcal{L}_{X^{3} \omega^{1}}+b_{2} \mathcal{L}_{X^{1}} \omega^{3}+c_{1} \mathrm{~d} i_{X^{3} \omega^{1}}+c_{2} \mathrm{~d} i_{X^{1}} \omega^{3}+e i_{X^{1}} i_{X^{3}} H\right) \text {. }
\end{aligned}
$$

The Leibniz rule of $A_{H}$ is equivalent to $\Omega=\Theta+\mathcal{T}$.

Putting $H=0$, we are in the situation of Theorem 2.7. Then by Theorem 2.7 (i.e., by Theorem 3.2 in [2]) we get $\left(b_{1}, b_{2}, c_{1}, c_{2}\right)=(0,0,0,0)$ or $\left(b_{1}, b_{2}, c_{1}, c_{2}\right)=(0, a, 0,0)$ or $\left(b_{1}, b_{2}, c_{1}, c_{2}\right)=(-a, a, 0,0)$ or $\left(b_{1}, b_{2}, c_{1}, c_{2}\right)=$ $(-a, a, a, 0)$. More, $A_{0}$ for such $\left(b_{1}, b_{2}, c_{1}, c_{2}\right)$ satisfies the Leibniz rule.

Therefore (as $c_{2}=0$ ) the Leibniz rule of $A_{H}$ is equivalent to the equality

$$
\begin{aligned}
& e a i_{X^{1}} i_{\left[X^{2}, X^{3}\right]} H+b_{2} e \mathcal{L}_{X^{1}} i_{X^{2}} i_{X^{3}} H \\
& =e a i_{\left[X^{1}, X^{2}\right]} i_{X^{3}} H+b_{1} e \mathcal{L}_{X^{3}} i_{X^{1}} i_{X^{2}} H+c_{1} e \mathrm{~d} i_{X^{3}} i_{X^{1}} i_{X^{2}} H \\
& \quad+e a i_{X^{2}} i_{\left[X^{1}, X^{3}\right]} H+b_{2} e \mathcal{L}_{X^{2}} i_{X^{1}} i_{X^{3}} H .
\end{aligned}
$$

If $\left(b_{1}, b_{2}, c_{1}, c_{2}\right)=(0,0,0,0)$, the above equality is equivalent to

$$
e a i_{X^{1}} i_{\left[X^{2}, X^{3}\right]} H=e a i_{\left[X^{1}, X^{2}\right]^{2}} i_{X^{3}} H+e a i_{X^{2}} i_{\left[X^{1}, X^{3}\right]} H .
$$

Putting $X^{1}=\partial_{1}, X^{2}=\partial_{1}+x^{1} \partial_{3}$ and $X^{3}=\partial_{2}$ we have $\left[X^{2}, X^{3}\right]=0$, $\left[X^{1}, X^{3}\right]=0$ and $\left[X^{1}, X^{2}\right]=\partial_{3}$, and then $0=e a i_{\partial_{3}} i_{\partial_{2}} H$ for any closed $H$ (for example for $H=\mathrm{d} x^{1} \wedge \mathrm{d} x^{2} \wedge \mathrm{d} x^{3}$ ). Consequently $e=0$ or $a=0$.

If $\left(b_{1}, b_{2}, c_{1}, c_{2}\right)=(0, a, 0,0)$, the above equality is equivalent to

$$
\begin{aligned}
& e a i_{X^{1}} i_{\left[X^{2}, X^{3}\right]} H+e a \mathcal{L}_{X^{1}} i_{X^{2}} i_{X^{3}} H \\
& =e a i_{\left[X^{1}, X^{2}\right]} i_{X^{3}} H+e a i_{X^{2}} i_{\left[X^{1}, X^{3}\right]} H+e a \mathcal{L}_{X^{2}} i_{X^{1}} i_{X^{3}} H .
\end{aligned}
$$

Putting $X^{1}=\partial_{1}, X^{2}=\partial_{2}$ and $X^{3}=\partial_{3}$ and $H=x^{2} \mathrm{~d} x^{1} \wedge \mathrm{d} x^{2} \wedge \mathrm{d} x^{3}$ (it is closed) we have $\left[X^{2}, X^{3}\right]=0,\left[X^{1}, X^{2}\right]=0,\left[X^{1}, X^{3}\right]=0, \mathcal{L}_{X^{2}} i_{X^{1}} i_{X^{3}} H=$ $\mathcal{L}_{\partial_{2}} x^{2} \mathrm{~d} x^{2}=\mathrm{d} x^{2}$ and $\mathcal{L}_{X^{1}} i_{X^{2}} i_{X^{3}} H=\mathcal{L}_{\partial_{1}}\left(-x^{2} \mathrm{~d} x^{1}\right)=0$. Then $e a \mathrm{~d} x^{2}=0$. So, $a=0$ or $e=0$.

If $\left(b_{1}, b_{2}, c_{1}, c_{2}\right)=(-a, a, 0,0)$, the above equality is equivalent to

$$
\begin{aligned}
& e a i_{X^{1}} i_{\left[X^{2}, X^{3}\right]} H+e a \mathcal{L}_{X^{1}} i_{X^{2}} i_{X^{3}} H \\
& \quad=e a i_{\left[X^{1}, X^{2}\right]} i_{X^{3}} H-e a \mathcal{L}_{X^{3}} i_{X^{1}} i_{X^{2}} H+e a i_{X^{2}} i_{\left[X^{1}, X^{3}\right]} H+e a \mathcal{L}_{X^{2}} i_{X^{1}} i_{X^{3}} H .
\end{aligned}
$$

Putting $X^{1}=\partial_{1}, X^{2}=\partial_{2}$ and $X^{3}=\partial_{3}$ and $H=x^{2} \mathrm{~d} x^{1} \wedge \mathrm{d} x^{2} \wedge \mathrm{d} x^{3}$ we have (see above) $\left[X^{2}, X^{3}\right]=0,\left[X^{1}, X^{2}\right]=0,\left[X^{1}, X^{3}\right]=0, \mathcal{L}_{X^{2}} i_{X^{1}} i_{X^{3}} H=\mathrm{d} x^{2}$, $\mathcal{L}_{X^{1}} i_{X^{2}} i_{X^{3}} H=0$ and $\mathcal{L}_{X^{3}} i_{X^{1}} i_{X^{2}} H=\mathcal{L}_{\partial_{3}}\left(-x^{2} \mathrm{~d} x^{3}\right)=0$. Then ead $x^{2}=0$. So, $a=0$ or $e=0$. 
If $\left(b_{1}, b_{2}, c_{1}, c_{2}\right)=(-a, a, a, 0)$, the above equality is equivalent to

$$
e a \sum\left\{i_{X^{1}} i_{\left[X^{2}, X^{3}\right]} H+\mathcal{L}_{X^{1}} i_{X^{2}} i_{X^{3}} H\right\}=e a \mathrm{~d} i_{X^{1}} i_{X^{2}} i_{X^{3}} H,
$$

where $\sum$ is the cyclic sum $\sum_{\operatorname{cycl}\left(X^{1}, X^{2}, X^{3}\right)}$. Then $e$ is arbitrary real number because from $\mathrm{d} H=0$ it follows

$$
\sum\left\{i_{X^{1}} i_{\left[X^{2}, X^{3}\right]} H+\mathcal{L}_{X^{1}} i_{X^{2}} i_{X^{3}} H\right\}=\mathrm{d} i_{X^{1}} i_{X^{2}} i_{X^{3}} H .
$$

Indeed, using $\mathrm{d} H=0$ and $i_{\left[X^{1}, X^{4}\right]}=\mathcal{L}_{X^{1}} i_{X^{4}}-i_{X^{4}} \mathcal{L}_{X^{1}}$ and the well-known formula expressing $\mathrm{d} H\left(X^{1}, X^{2}, X^{3}, X^{4}\right)$, we have

$$
\begin{gathered}
\sum\left\{i_{X^{4}} i_{X^{1}} i_{\left[X^{2}, X^{3}\right]} H+i_{X^{4}} \mathcal{L}_{X^{1}} i_{X^{2}} i_{X^{3}} H\right\} \\
=\sum\left\{i_{X^{4}} i_{X^{1}} i_{\left[X^{2}, X^{3}\right]} H+\mathcal{L}_{X^{1}} i_{X^{4}} i_{X^{2}} i_{X^{3}} H-i_{\left[X^{1}, X^{4}\right]} i_{X^{2}} i_{X^{3}} H\right\} \\
=6 \sum\left\{H\left(\left[X^{2}, X^{3}\right], X^{1}, X^{4}\right)+X^{1} H\left(X^{3}, X^{2}, X^{4}\right)\right. \\
\left.-H\left(X^{3}, X^{2},\left[X^{1}, X^{4}\right]\right)\right\} \\
=-24 \mathrm{~d} H\left(X^{1}, X^{2}, X^{3}, X^{4}\right)+6 X^{4} H\left(X^{3}, X^{2}, X^{1}\right)=i_{X^{4}} \mathrm{~d} i_{X^{1}} i_{X^{2}} i_{X^{3}} H .
\end{gathered}
$$

Summing up, given a real number $a \neq 0$ we have $\left(b_{1}, b_{2}, c_{1}, c_{2}, e\right)=$ $(0,0,0,0,0)$ or $\left(b_{1}, b_{2}, c_{1}, c_{2}, e\right)=(0, a, 0,0,0)$ or $\left(b_{1}, b_{2}, c_{1}, c_{2}, e\right)=$ $(-a, a, 0,0,0$,$) or \left(b_{1}, b_{2}, c_{1}, c_{2}, e\right)=(-a, a, a, 0, e)$, where $e$ may be arbitrary real number. If $a=0$ we have $\left(b_{1}, b_{2}, c_{1}, c_{2}, e\right)=(0,0,0,0, e)$, where $e$ may be arbitrary. Theorem 5.3 is complete.

Corollary 5.4. If $m \geq 3$, then the twisted Courant bracket $\mathcal{M} f_{m}$-natural operator from Example 3.2 is the unique $\mathcal{M} f_{m}$-natural operator $A$ in the sense of Definition 3.1 for $p=3$ satisfying the following conditions:

(C1) $A_{H}\left(\rho_{1}, A_{H}\left(\rho_{2}, \rho_{3}\right)\right)=A_{H}\left(A_{H}\left(\rho_{1}, \rho_{2}\right), \rho_{3}\right)+A_{H}\left(\rho_{2}, A_{H}\left(\rho_{1}, \rho_{3}\right)\right)$,

(C2) $A_{H}(X \oplus 0, Y \oplus 0)=[X, Y] \oplus i_{X} i_{Y} H$

for all $\rho_{1}, \rho_{2}, \rho_{3}, X \oplus 0, Y \oplus 0 \in \mathcal{X}(M) \oplus \Omega^{1}(M)$, all closed $H \in \Omega_{c l}^{3}(M)$ and all $m$-manifolds $M$.

Proof. Indeed, the condition $(C 1)$ and Theorem 5.3 imply that $A=A^{\langle 1, a\rangle}$ or $A=A^{\langle 2, a\rangle}$ or $A=A^{\langle 3, a\rangle}$ or $A=A^{\langle 4, a, e\rangle}$ for some real numbers $a$ and $e$. Then $(C 2)$ implies that $A=A^{\langle 4, a, e\rangle}$ and $a=1$ and $e=1$ because $A_{H}^{\langle 1, a\rangle}(X \oplus$ $0, Y \oplus 0)=a[X, Y] \oplus 0$ and $A_{H}^{\langle 2, a\rangle}(X \oplus 0, Y \oplus 0)=a[X, Y] \oplus 0$ and $A_{H}^{\langle 3, a\rangle}(X \oplus$ $0, Y \oplus 0)=a[X, Y] \oplus 0$ and $A_{H}^{\langle 4, a, e\rangle}(X \oplus 0, Y \oplus 0)=a[X, Y] \oplus e i_{X} i_{Y} H$.

Corollary 5.5. If $m \geq 3$, any $\mathcal{M} f_{m}$-natural operator $A$ in the sense of Definition 3.1 for $p=3$ such that $A_{H}$ is a Lie algebra bracket (i.e., it is skewsymmetric, bilinear and satisfying the Leibniz rule) for all closed 3-forms $H \in \Omega_{c l}^{3}(M)$ and all $m$-manifolds $M$ is one of the $\mathcal{M} f_{m}$-natural operators:

$$
\begin{array}{rlrl}
A_{H}^{\langle 1, a\rangle}\left(\rho_{1}, \rho_{2}\right) & = & a\left[X^{1}, X^{2}\right] \oplus 0, \\
A_{H}^{\langle 2, a\rangle}\left(\rho^{1}, \rho^{2}\right) & =a\left[X^{1}, X^{2}\right] \oplus\left(a\left(\mathcal{L}_{X^{1}} \omega^{2}-\mathcal{L}_{X^{2}} \omega^{1}\right)\right), \\
A_{H}^{\langle 4,0, e\rangle}\left(\rho^{1}, \rho^{2}\right) & = & 0 \oplus e i_{X^{1}} i_{X^{2}} H,
\end{array}
$$

where $\rho^{1}=X^{1} \oplus \omega^{1}$ and $\rho^{2}=X^{2} \oplus \omega^{2}$, and a and $e$ are arbitrary real numbers. 
Proof. It follows from Theorem 5.3.

Corollary 5.6. If $m \geq 3$, any $\mathcal{M} f_{m}$-natural operator $A$ in the sense of Definition 3.1 for $p=3$ satisfying the Leibniz rule for all 3 -forms $H$ (or for all closed 3-forms and at least one non-closed 3-form) is one of the $\mathcal{M} f_{m}$-natural operators:

$$
\begin{aligned}
& A_{H}^{\langle 1, a\rangle}\left(\rho_{1}, \rho_{2}\right)=\quad a\left[X^{1}, X^{2}\right] \oplus 0, \\
& A_{H}^{\langle 2, a\rangle}\left(\rho^{1}, \rho^{2}\right)=a\left[X^{1}, X^{2}\right] \oplus\left(a\left(\mathcal{L}_{X^{1}} \omega^{2}-\mathcal{L}_{X^{2}} \omega^{1}\right)\right), \\
& A_{H}^{\langle 3, a\rangle}\left(\rho^{1}, \rho^{2}\right)=\quad a\left[X^{1}, X^{2}\right] \oplus\left(a \mathcal{L}_{X^{1}} \omega^{2}\right), \\
& A_{H}^{\langle 4, a, 0\rangle}\left(\rho^{1}, \rho^{2}\right)=a\left[X^{1}, X^{2}\right] \oplus\left(a\left(\mathcal{L}_{X^{1}} \omega^{2}-i_{X^{2}} \mathrm{~d} \omega^{1}\right)\right) \text {, } \\
& A_{H}^{\langle 4,0, e\rangle}\left(\rho^{1}, \rho^{2}\right)=\quad 0 \oplus e i_{X^{1}} i_{X^{2}} H,
\end{aligned}
$$

where $\rho^{1}=X^{1} \oplus \omega^{1}$ and $\rho^{2}=X^{2} \oplus \omega^{2}$, and a and $e$ are arbitrary real numbers.

Proof. It follows from Theorem 5.3 and its proof.

Remark 5.7. It is well-known that given closed 3-form $H \in \Omega_{c l}^{3}(M)$ on a $m$-manifold $M$, the twisted Courant bracket $[-,-]_{H}:\left(\mathcal{X}(M) \oplus \Omega^{1}(M)\right) \times$ $\left(\mathcal{X}(M) \oplus \Omega^{1}(M)\right) \rightarrow \mathcal{X}(M) \oplus \Omega^{1}(M)$ is bilinear and satisfies the properties (A1)-(A5) from Corollary 2.8 for all $\rho_{1}, \rho_{2}, \rho_{3} \in \mathcal{X}(M) \oplus \Omega^{1}(M)$ and all $f \in \mathcal{C}^{\infty}(M)$, see $[3,8]$, but $[-,-]_{H} \neq[-,-]_{0}$ if $H \neq 0$. Is it a contradiction with the uniqueness from Corollary 2.8? No, it is not. Indeed, $[-,-]_{H}$ is not extendable to a $\mathcal{M} f_{m}$-natural bilinear operator in the sense of Definition 2.1 because it is invariant only with respect to $\mathcal{M} f_{m}$-maps $\varphi: M \rightarrow M$ preserving $H$, in fact.

Remark 5.8. By Corollary 5.5, given a closed 3-form $H$ on $M$, the skewsymmetric bracket $\left[\left[X^{1} \oplus \omega^{1}, X^{2} \oplus \omega^{2}\right]\right]^{(H)}:=0 \oplus i_{X^{1}} i_{X^{2}} H$ satisfies the Leibniz rule. One can easily directly verify that $\left(T M \oplus T^{*} M, e[[-,-]]^{(H)}, 0 \pi\right)$ for arbitrary fixed $e \in \mathbf{R}$ and closed 3 -form $H$ is a Lie algebroid canonically depending on $H$. So, if we have a closed 3 -form $H$ on a $m$-manifold $M$, we can construct canonical (in $H)$ Lie algebroids $\left(E M,[[-,-]]^{[H]}, a^{[H]}\right)$ with $E M=T M \oplus T^{*} M$ different than the one from Proposition 2.11.

\section{Acknowledgements}

I would like to thank the reviewer for valuable suggestions. By one of them I was inspired to study the problem given in Proposition 2.11.

Open Access. This article is distributed under the terms of the Creative Commons Attribution 4.0 International License (http://creativecommons.org/licenses/by/4. 0/), which permits unrestricted use, distribution, and reproduction in any medium, provided you give appropriate credit to the original author(s) and the source, provide a link to the Creative Commons license, and indicate if changes were made.

Publisher's Note Springer Nature remains neutral with regard to jurisdictional claims in published maps and institutional affiliations. 


\section{References}

[1] Courant, T.: Dirac manifolds. Trans. Am. Math. Soc. 319(631), 631-661 (1990)

[2] Doupovec, M., Kurek, J., Mikulski, W.M.: The natural brackets on couples of vector fields and 1-forms. Turk. J. Math. 42(2), 1853-1862 (2018)

[3] Gualtieri, M.: Generalized complex geometry. Ann. Math. 174(1), 75-123 (2011)

[4] Hitchin, N.: Generalized Calabi-Yau manifolds. Q. J. Math. 54(3), 281-308 (2003)

[5] Kolář, I., Michor, P.W., Slovák, J.: Natural Operations in Differential Geometry. Springer, Berlin (1993)

[6] Mackenzie, K.C.H.: General Theory of Lie Groupoids and Lie Algebroids. London Math. Soc., Lecture Note 213. Cambridge University Press, Cambridge (2005)

[7] Liu, Z.J., Weinstein, A., Xu, P.: Main triples for Lie bialgebroids. J. Differ. Geom. 45, 547-574 (1997)

[8] Ševera, P., Weinstein, A.: Poisson geometry with a 3-form bacground. Progr. Teoret. Phys. Suppl., 145-154 (2001)

Włodzimierz M. Mikulski

Institute of Mathematics

Jagiellonian University

ul. Łojasiewicza 6

30-348 Kraków

Poland

e-mail: Wlodzimierz.Mikulski@im.uj.edu.pl

Received: January 24, 2019.

Revised: February 11, 2019.

Accepted: June 10, 2019. 